\title{
Estudio Ergonómico en la Unidad de Sala de Partos del Hospital Regional de Talca
}

\author{
ERGONOMIC STUDY IN THE DELIVERY ROOM UNIT OF THE REGIONAL HOSPITAL OF TALCA
}

\author{
Valeria Paz Bravo Carrasco
}

Universidad de Concepción

\section{RESUMEN}

El presente estudio se orientó a analizar la Unidad de Sala de Pre-partos/ partos, la cual forma parte de una de las seis áreas que componen la Unidad de Obstetricia y Ginecología del Hospital Regional de Talca. El puesto se basa principalmente en el desarrollo sistémico de entrada, salida del personal y su estancia en el desarrollo de la actividad, debido a la exigencia laboral que presenta esta en cuanto a demandas físicas, psicológicas y organizacionales dentro del área de trabajo.

$\mathrm{Al}$ ser considerada una unidad de urgencia, se caracteriza por presentar la mayor complejidad dentro del servicio, debido a que se evidencian casos de alto riesgo de pacientes y, por otro lado, situaciones programadas en el caso de cesáreas electivas e inducciones de parto.

El levantamiento realizado permitió hallar factores de tiempos de trabajo y actividades, físicos (carga física, sobrecarga postural) y mentales (carga mental y factores psicosociales). Estos factores fueron evaluados por una lista de verificación, descripción de actividades, porcentaje de carga cardiovascular, evaluación de mobiliario de trabajo y medidas antropométricas, RULA, cuestionario de Pittsburgt (calidad del sueño) y la aplicación de la encuesta de riesgos psicosociales SUSESO-ISTAS 21. Se concluye que, al ir en aumento el porcentaje de pacientes que ingresan al servicio en situaciones programadas y de urgencia, se dificulta su atención en las unidades por falta de camas disponibles, complicación en su traslado y estadía en ellas. Por ello, se recomiendan soluciones que disminuyan la carga de trabajo y mejoren el ambiente laboral.

(Bravo V, 2018. Estudio Ergonómico en la Unidad de Sala de Partos del Hospital Regional de Talca. Cienc Trab. Ene-Abr; 20 [61]: 48-55).

Palabras claves: POSTURA, ATENCIÓN DE PACIENTES, ESTRÉS LABORAL.

\section{ABSTRACT}

The present study was oriented to analyze the Unit of Labor/Delivery Room, which is part of one of the six areas that make up the Obstetrics and Gynecology Unit of the Regional Hospital of Talca. The position is mainly based on the systemic development of entry, exit of staff and their stay in the development of the activity, due to the labor requirement that this presents in terms of physical, psychological and organizational demands within the work area.

As an emergency unit, it is characterized by presenting the greatest complexity within the service, due to the fact that there are cases of high risk of patients and, on the other hand, situations programmed in the case of elective cesareans and labor induction.

The data collected made possible to find factors of work times and activities, physical (physical load, postural overload) and mental (mental load and psychosocial factors). These factors were evaluated by a checklist, description of activities, percentage of cardiovascular load, evaluation of work furniture and anthropometric measurements, RULA, Pittsburgt questionnaire (quality of sleep) and the application of the psychosocial risk survey SUSEO-ISTAS twenty-one.

It is concluded that, as the percentage of patients entering the service in programmed and urgent situations increases, it becomes difficult for them to attend to the units due to the lack of available beds, complication in their transfer and stay in them. Therefore, solutions that reduce the workload and improve the work environment are recommended.

Key words: POSTURE, PATIENT CARE, WORK STRESS.

\section{INTRODUCCIÓN}

El Hospital de Talca César Garavagno Burotto, fue fundado en 1803 y se encuentra ubicado en Chile, en la séptima región del Maule de la ciudad de Talca; el establecimiento hospitalario se encuentra inserto en una estructura basada en redes, en donde cada uno de sus

\section{Correspondencia / Correspondence:}

Valeria Páz Bravo

3 oriente \# 1015. Edificio Alameda Talca, Chile

e-mail:vbravocarrasco@gmail.com.

Recibido: 25 de Noviembre de 2016 / Aceptado: 28 de Diciembre de 2017 integrantes contribuye al logro de los objetivos sanitarios. Por otro lado, cumple funciones como hospital regional, provincial y comunal, siendo el centro de referencia para los hospitales de la región. Es por esto que, dentro de este centro asistencial, se presenta una dotación de 1.468 cargos y 603 camas (compuesta por 471 camas básicas y 132 camas de atención a pacientes críticos), comprende una organización de cuatro servicios clínicos básicos como pediatría, medicina, cirugía, obstetricia y ginecología y siete servicios clínicos de especialidades como urología, traumatología, oftalmología, otorrinolaringología, psiquiatría, cirugía infantil y neonatología.

Además, presenta unidades de apoyo terapéutico y diagnóstico (diálisis, centro de cáncer gástrico, unidad de oncología ambulatoria, unidad de anatomía patológica y 10 pabellones quirúrgicos equipados, de los cuales 9 se encuentran centralizados en la placa técnica y uno en dependencias del servicio de Obstetricia y Ginecología). Cuenta con instalaciones para la atención de 
pacientes críticos adultos, pediátricos y neonatales; estos últimos, únicos en la región, incluyendo el sector privado. Destaca también la unidad de nutrición parenteral, la cual cuenta con la más moderna tecnología del país en esta materia. ${ }^{1}$ El hospital, se encuentra establecido por un organismo administrador de la ley 18.834 del estatuto administrativo, ley 19.937/2004 de autoridad sanitaria, ley 15.076 médica de contrato 28 horas y ley 19.664 médica de contrato 22 horas. Por su parte, presenta una estructura organizacional matricial, compuesta por la dirección con unidades de staff, unidades asesoras coordinadoras, seis subdirecciones y quince centros de responsabilidad. ${ }^{2}$

Dentro de las áreas que componen el hospital, se encuentra el servicio de Obstetricia y ginecología, el cual corresponde a la unidad de maternidad que brinda cuidados y atención de mediana y alta complejidad a la mujer durante todo su ciclo vital en las áreas de Ginecología General, Onco-ginecología, Patología Mamaria, Alto Riesgo Obstétrico, Atención de Pre-parto, parto y puerperio, así como también al recién nacido durante el parto y en su estadía en alojamiento conjunto con su madre.

El tema de investigación del presente estudio se basa en determinar las características del trabajo del subsistema de Obstetricia y Ginecología en la unidad de sala de pre-partos/partos, considerada como unidad de atención de urgencia, la cual está enfocada a las usuarias que acceden a recibir la atención de la unidad de maternidad y la cuantificación de los niveles de demandas físicas, psicológicas y organizacionales dentro del área de trabajo a las que se ven expuestas el personal en su jornada de trabajo. A esto se agrega el estudio de variables relacionadas con la carga física, análisis de mobiliario y de sueño como una forma de evaluar su calidad de vida laboral en el desempeño de su sistema de trabajo en cuarto turno.

\section{Objetivos}

Objetivo General

- Detectar factores de riesgo en la unidad de sala de pre-partos/ partos y como estos inciden en su resultado a nivel organizacional en la calidad de vida laboral dentro del subsistema de Obstetricia y Ginecología.

\section{Objetivos Específicos}

- Determinar las características organizacionales y de trabajo en la unidad de pre-partos/partos del Hospital de Talca.

- Evaluar la carga física a la que están expuestos los trabajadores de la unidad de pre-partos/partos .

- Conocer las condiciones generadas en cada situación (programada y urgencia) de atención de partos y los riesgos psicosociales expuestos en el grupo de trabajo de la unidad.

- Conocer las condiciones de equipamiento en el cual se desarrolla el trabajo en la unidad de pre-partos/partos .

- Determinar la calidad del sueño que presenta la unidad de prepartos/partos, en su sistema de cuarto turno.

- Proponer medidas de mejoramiento de condiciones de trabajo para las personas expuestas.

\section{MATERIALES Y MÉTODOS}

El presente estudio enfoca sus objetivos en el desarrollo del proceso de atención da la unidad pre-partos/partos y el impacto que genera en la unidad obstétrica y ginecológica de sus áreas que la conforman.
Tabla 1.

Lista de chequeo. Aspectos considerados en la lista de chequeo de levantamiento de factores ergonómicos en un puesto de trabajo.

$\begin{array}{ll}\text { Unidad } & \text { Aspectos a Considerar } \\ \text { Organización del trabajo } & \text { Aumento del flujo de pacientes } \\ & \text { Turnos de trabajo } \\ & \text { Procedimientos } \\ & \text { Comunicación } \\ & \text { Pausas o descansos programados } \\ & \text { Días libres, tiempo extra } \\ & \text { Puesto y espacio de trabajo } \\ \text { Carga física } & \text { Posturas } \\ & \text { Antropometría } \\ & \text { Trabajo repetitivo } \\ \text { Carga ambiental } & \text { Ruído } \\ & \text { Iluminación } \\ & \text { Calor } \\ \text { Carga mental } & \text { Descanso } \\ & \text { Sincronización } \\ & \text { Ritmo } \\ & \text { Estrés laboral } \\ & \text { Responsabilidad / riesgo } \\ \text { Carga psicológica } & \text { Presión por la urgencia de acuerdo a } \\ & \text { las patologías que traen las pacientes. } \\ & \text { Avance de trabajo de parto } \\ & \text { Aumento del flujo de pacientes }\end{array}$

Tabla 2.

Listado de materiales.

$\begin{array}{ll}\text { Material } & \text { Objetivo } \\ \text { Cinta métrica } & \begin{array}{l}\text { Determinar dimensiones del puesto de trabajo, } \\ \text { distancias, alcances de los elementos de trabajo y } \\ \text { las distancias del trabajador. } \\ \text { Peso corporal del trabajador. }\end{array} \\ \begin{array}{ll}\text { Balanza } & \text { Toma de ángulos durante la dinámica de trabajo } \\ \text { Goniómetro } & \text { (imágenes de postura y alcances) de la matrona. } \\ \text { Cronómetro } & \begin{array}{l}\text { Identificar tiempo de trabajo efectivo (cronometraje } \\ \text { con vuelta a cero). }\end{array} \\ \text { Polar PM 26 Beurer } & \begin{array}{l}\text { Determinar la FC de reposo y trabajo, obteniendo el } \\ \text { \% CC por cada una hora de trabajo durante una } \\ \text { jornada del turno (12 hrs.). }\end{array} \\ \text { Hoja de registro de tiempo } & \begin{array}{l}\text { Registro del tiempo y actividad según horario de } \\ \text { trabajo. } \\ \text { Cámara fotográfica }\end{array} \\ \text { Toma de imágenes durante el desarrollo de la } \\ \text { actividad. } \\ \text { Construcción del informe y suministro de información. } \\ \text { Lista de chequeo }\end{array} \\ \begin{array}{l}\text { Determinar riesgos ergonómicos en forma cualitativa en } \\ \text { la actividad de atención de partos (diagnóstico inicial). }\end{array}\end{array}$

Por esto, se realiza lo siguiente:

a. Caracterización de actividades: se realiza una lista de verificación y una secuencia descriptiva de todas las actividades efectuadas para establecer un análisis de una matrona.

b. Carga de trabajo: Se someterá a evaluación a una matrona para obtener su frecuencia cardiaca mediante la utilización de un polar, para obtener el porcentaje de carga cardiovascular del funcionario y determinar si la dinámica de trabajo presenta una carga física. Esta se obtiene en una jornada de trabajo (turno de $12 \mathrm{hrs}$.).

c. Caracterización de mobiliario: Se realiza un análisis sobre las condiciones del mobiliario que se encuentra en la unidad y trastornos músculo-esqueléticos asociados a su utilización, por lo que se considera las dimensiones antropométricas de la matrona evaluada.

d. Sobrecarga postural: Se aplica el método RULA, el cual evalúa la exposición de los trabajadores a factores de riesgo que pueden 
ocasionar trastornos en los miembros superiores del cuerpo: posturas, repetitividad de movimientos, fuerzas aplicadas, actividad estática del sistema músculo-esquelético. ${ }^{3}$

e. Calidad del sueño: Se aplicó el cuestionario de Pittsburgh con el objetivo de evaluar el nivel de sueño y sus efectos. ${ }^{4}$

f. Nivel de carga mental: Se realiza ISTAS 21 al turno del equipo de trabajo de la unidad de pre-partos/partos (un total de 9 personas), para determinar los riesgos psicosociales presentes en esta y como afectan en su desarrollo de funciones. ${ }^{5}$

\section{RESULTADOS Y DISCUSIÓN}

\section{Desarrollo}

\section{Análisis general del sistema de trabajo}

El Hospital de Talca es un organismo de carácter regional en la región del Maule que recibe, atiende, administra la salud de las personas y forma parte de la red asistencial de pacientes que son derivadas de los centros de menor complejidad. El sistema general del Hospital es el siguiente:

Figura 1. Distribución de áreas Hospital de Talca (sistema).

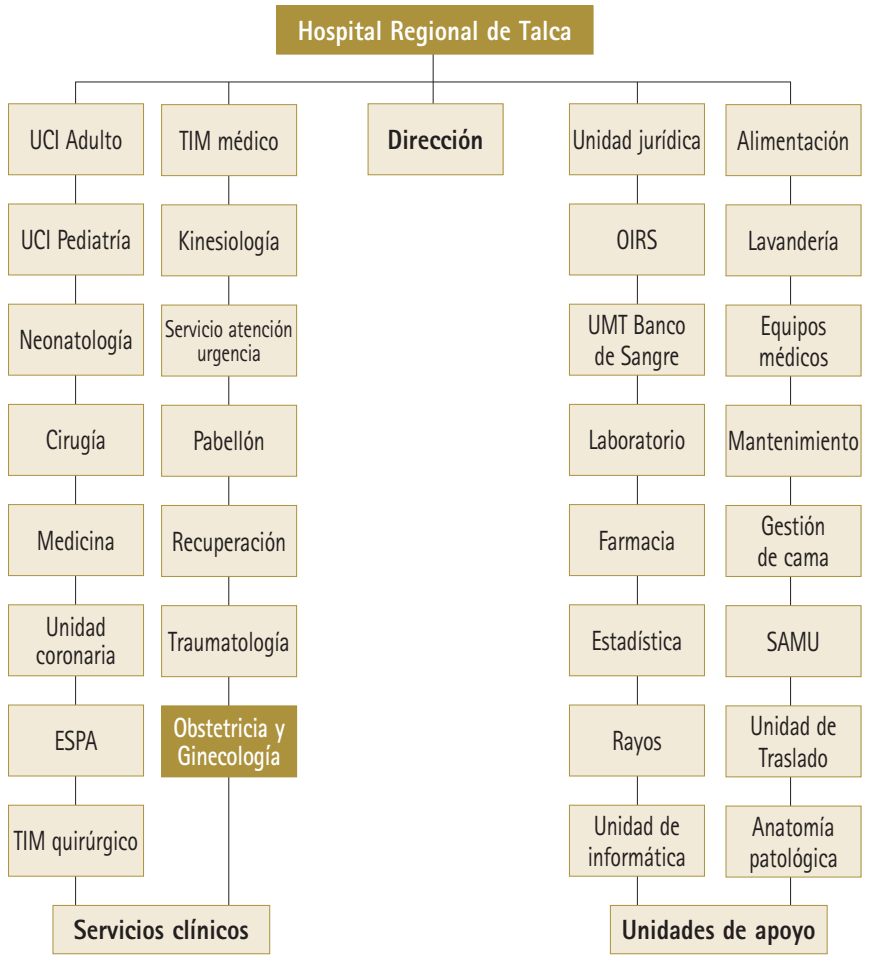

El sistema Hospital de Talca cuenta con el subsistema de Obstetricia y Ginecología, el cual está conformado por las siguientes unidades:

Figura 2. Distribución del subsistema.

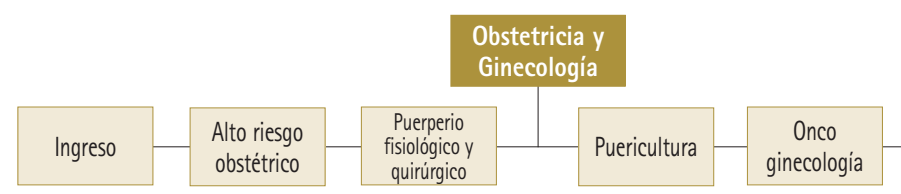

Pre-partos/partos

La caracterización de las actividades se realizó mediante la observación de estas desde el ingreso de la paciente hasta el alta a su domicilio, desde una actividad programada (inducciones de parto y cesáreas electivas) y una de urgencia (atención de parto, crisis hipertensiva, frenación de trabajo de parto prematuro, metrorragias que pueden ser producidas por placenta previa, desprendimiento de placenta, etc.) lo que se refleja directamente en la salud del personal. 
Figura 3.

Situación programada.

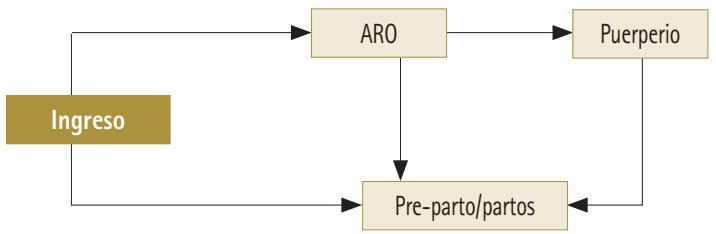

Tabla 3.

Descripción situación programada en sala pre-partos/parto.

\begin{tabular}{|c|c|}
\hline Programada & Descripción \\
\hline $\begin{array}{l}\text { 1. La paciente llega a la unidad de } \\
\text { ingreso y de ahi es enviada a alto } \\
\text { riesgo obstétrico (ARO). }\end{array}$ & $\begin{array}{l}\text { Todas las pacientes con patología de } \\
\text { embarazo son referidas a esta unidad. } \\
\text { Las pacientes con indicación de inducción de } \\
\text { parto se avisa durante la mañana y resto del } \\
\text { día según disponibilidad de camas en } \\
\text { pre-partos. Las cesárea electiva son avisadas } \\
\text { del día anterior. }\end{array}$ \\
\hline $\begin{array}{l}\text { 2. Desde ingreso la paciente puede } \\
\text { ser enviada a puerperio. }\end{array}$ & $\begin{array}{l}\text { Cuando no hay disponibilidad de cama en } \\
\text { ARO, la paciente queda en puerperio. }\end{array}$ \\
\hline $\begin{array}{l}\text { 3. Pre-partos/partos recibe pacientes } \\
\text { desde ARO y puerperio. }\end{array}$ & Según disponibilidad de camas. \\
\hline $\begin{array}{l}\text { 4. Post-parto inmediato ( } 2 \text { hrs.) la } \\
\text { paciente es enviada a la unidad de } \\
\text { puerperio donde permanecerá } \\
\text { durante } 48 \text { hrs. para ser dada de } \\
\text { alta a su domicilio. }\end{array}$ & $\begin{array}{l}\text { El traslado se realiza con el auxiliar de senvicio } \\
\text { y una técnico paramédico, a través de un } \\
\text { pasillo y luego una manga que presenta } \\
\text { desniveles en su recorrido. }\end{array}$ \\
\hline
\end{tabular}

Figura 4.

Situación de urgencia.

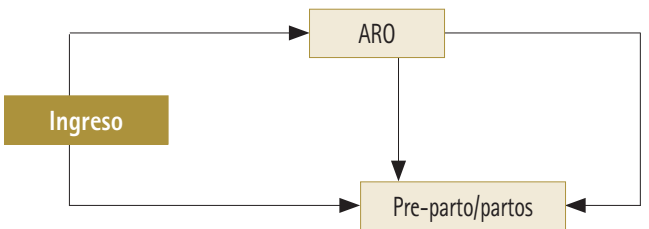

Tabla 4.

Descripción situación de urgencia en la sala pre-partos/parto.

$\begin{array}{ll}\text { Urgencia } & \text { Descripción } \\ \begin{array}{ll}\text { 1. La paciente llega desde ingreso } \\ \text { a la unidad de pre-partos/partos. }\end{array} & \begin{array}{l}\text { La paciente es recibida. Si no hay cama } \\ \text { disponible, permanece en un carro de } \\ \text { traslado en pre-parto. }\end{array} \\ \begin{array}{ll}\text { 2. Desde ARO también se trasladan } \\ \text { pacientes de urgencia, previo }\end{array} & \begin{array}{l}\text { Se recibe y se atiende de acuerdo a la } \\ \text { disponibilidad de cama o si no permanece } \\ \text { aviso telefónico. }\end{array}\end{array}$

Resumiendo: anteriormente se establece que en situaciones programadas y de urgencias se presenta un aumento de pacientes en las unidades, lo que produce sobrecarga en el trabajo. Además, se genera un aumento de traslados de paciente. Por otro lado, la afluencia de pacientes predispone la reducción de camas disponibles en las unidades, por lo cual la paciente debe ubicarse en un carro hasta que se presente disponibilidad (ARO y pre-parto/parto).

\section{Caracterización de la actividad}

Para la recolección de datos se consideró la participación de una matrona permanente en la unidad de pre-partos/partos.
Secuencia y descripción de las actividades desarrolladas en la unidad de pre-partos/parto (urgencia)

Tabla 6. Análisis de actividades en urgencia.

Tarea

1. Ingreso de la Se revisa la ficha clínica de la paciente, se controlan paciente a la unidad los signos vitales, se hace un examen físico y pre-partos/partos

obstétrico, se revisa la identificación (brazalete con nombre y rut). Luego se cumplen indicaciones médicas, si tiene dilatación avanzada se ofrece anestesia peridural e instalación de vía venosa. Se realiza un control de dinámica uterina (se controla cada 1 hora) y latidos cardio-fetales (se controla cada 30 minutos), se instala monitor fetal.

2. Controles de Se controlan las contracciones de la paciente en dinámica uterina y 10 minutos y se anotan los latidos cada 30 minutos. latidos cardio-fetales.

\section{Tacto vaginal}

Primero informar a la paciente del procedimiento. Lavado de manos, colocarse el guante estéril. Se procede a realizar el tacto vaginal previo aseo perineal realizado por la técnico paramédico. Se realiza el tacto vaginal. Posteriormente, se realiza aseo y se deja cómoda la paciente y se le informa el resultado del procedimiento. Se puede controlar hasta 5 veces.

4. Atención Primero se debe preparar a la paciente, poniéndola del parto en posición de parto (posición ginecológica) y semisentada. Lavado quirúrgico de manos, se viste a la paciente con ropa estéril y se pincela la zona genital para recibir el bebé. Se educa a la paciente en la forma que debe respirar para poder pujar. Se espera hasta que se produzca el parto, se recibe el recién nacido, se pinza el cordón y el recién nacido lo recibe la matrona de recién nacido, dejándolo en apego a la paciente. Se espera que se produzca el alumbramiento (salida de la placenta) y una vez ocurrido esto se verifica si ha habido desgarro, se debe proceder a suturar el periné, si es necesario se sutura, después se realiza aseo a la paciente y se deja en posición cómoda, se ordena el material usado. Se procede a realizar todos los registros. La paciente permanece en sala de parto por 2 horas realizando un control horario. En el segundo control se traslada a puerperio.

5. Registros Se procede a realizar confección de fichas, registro estadístico, recetas de insumos utilizados, comprobante de parto.

6. Control Se observa el sangramiento y la retracción

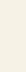

7. Traslado Posterior al segundo control, se verifica que la 2 días. a puerperio paciente está controlada, se traslada a puerperio por una estancia de 2 días para posterior alta a su domicilio.

Nota: En situaciones programadas el procedimiento es el mismo, a diferencia que se reciben pacientes para inducción de parto y cesárea electiva, las cuales no siempre se efectúan dentro del horario del turno, ya que pueden presentar una estancia de 2 hasta 3 días en espera de su parto.

Tabla 5.

Datos funcionario evaluado.

\begin{tabular}{|cc|}
\hline Antecedentes & Trabajador 1 \\
\hline Edad & 60 años \\
Talla & $168 \mathrm{~cm}$. \\
Peso & $80 \mathrm{Kg}$. \\
IMC & 28,3 \\
Permanencia & 38 años \\
\hline
\end{tabular}


Figura 5.

Ingreso a sala de pre-partos/parto.
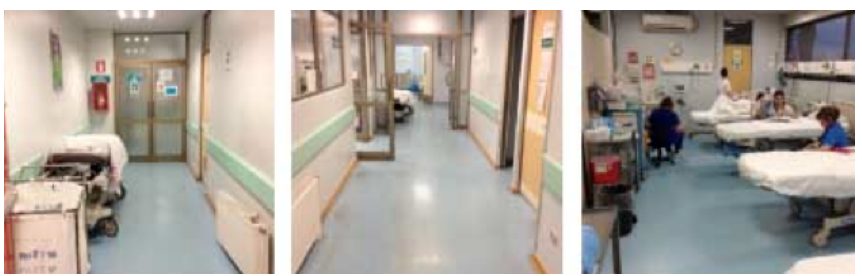

Figura 6.

Zona de registros / Recuperación de anestecia.
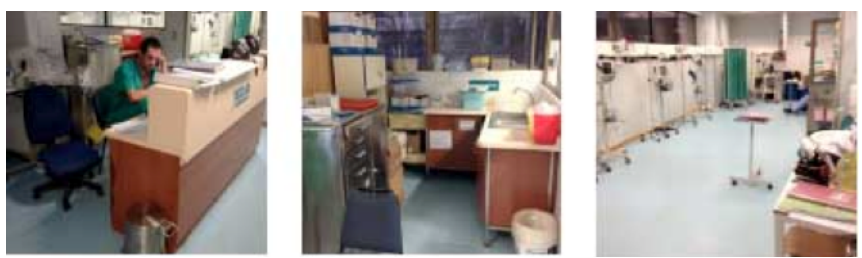

Figura 7.

Zona de descanso y alimentación / Unidad de atención inmediata.
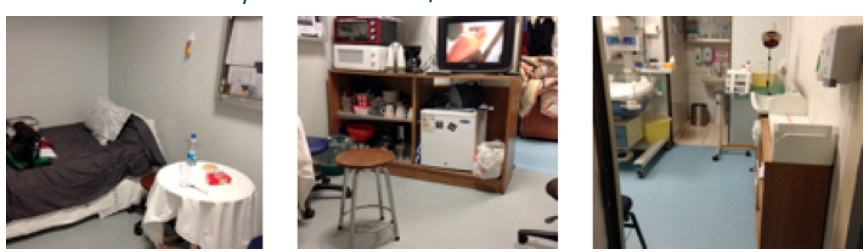

\section{Carga de trabajo}

Se realizó la evaluación de frecuencia cardiaca $(\mathrm{FC})$ en la matrona evaluada, en donde su frecuencia de reposo fue de 73 lat $/ \mathrm{min}$. El proceso de obtención de la frecuencia de trabajo fue cada una hora, efectuando un estudio en terreno durante un jornada de trabajo de un turno de 12 horas (de 8:00 am. a 20:00 pm.). La determinación de la carga física, se efectúa mediante la utilización de un Polar, obteniendo los siguientes resultados:

Tabla 7.

Promedio por hora y \%CC en matrona evaluada.

$\begin{array}{lcc}\text { TIEMPO } & \text { FC Trabajo } & \% \text { CC } \\ \text { H1 } & 75 & 2,29 \\ \text { H2 } & 68 & 5,74 \\ \text { H3 } & 63 & 11,49 \\ \text { H4 } & 84 & 12,64 \\ \text { H5 } & 94 & 24,1 \\ \text { H6 } & 90 & 19,54 \\ \text { H7 } & 26,43 \\ \text { H8 } & 96 & 25,28 \\ \text { H9 } & 95 & 19,54 \\ \text { H10 } & 90 & 16,09 \\ \text { H11 } & 87 & 12,64 \\ \text { H12 } & 84 & 24,13 \\ & 94 & \text { \% CC jornada: } 16,66\end{array}$

Los datos obtenidos en \% CC en las horas de trabajo evaluadas demuestran que no hay riesgo de exposición de un trabajo pesado, debido a que la actividad que desempeña la funcionaria no presenta una demanda física elevada, es decir, se efectúa un promedio de la jornada de 16,66 \% CC. El trabajo se relaciona de forma directamente con la paciente, se presenta una atención personalizada y no se genera un esfuerzo físico mayor para la atención de esta. Sin embargo, el factor estrés puede aumentar la FC en situaciones que se presente una mayor complejidad en la atención o urgencia de esta. En este caso se hace referencia a la H7, H8 y H12 donde su carga cardiovascular fue más alta alcanzando valores desde $24 \%$ a $26 \%$ debido a que en ese momento la funcionaria se encuentra realizando una atención de parto. Si bien la H12 al ser el último momento de trabajo, la funcionaria debe acudir a la atención del parto, la cual efectúa un alza en su porcentaje de carga cardiovascular.

Tanto en los eventos programados como en los eventos de urgencia se ven impactados por la afluencia de pacientes a la unidad. Cuando esto sucede, el problema que se genera es que la carga de trabajo aumenta durante del transcurso de la jornada.

\section{Caracterización de mobiliario y del usuario \\ Análisis antropométrico y de mobiliario}

Se realiza la interacción de la matrona en relación al equipamiento utilizado al momento de atender un parto (Fig. 3), conformando un sistema funcional entre ambos. Por esto, se detectan falencias significativas en relación a las características propias del mobiliario en relación al personal de atención.

\section{Figura 8.}

Matrona y utilización de mobiliario en pre-partos/parto (mesa de parto, asiento, camilla y funcionaria evaluada).
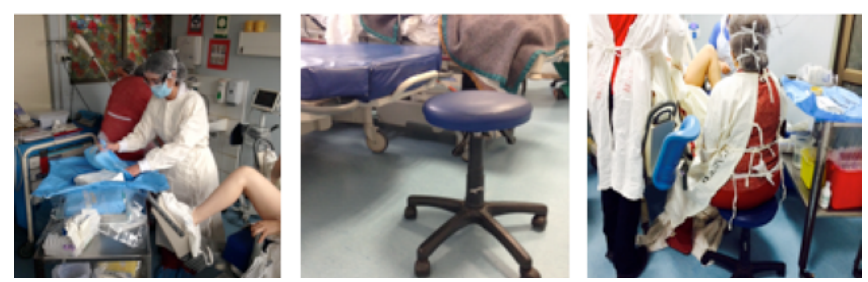

\section{Análisis antropométrico}

En las dimensiones que se presentan a continuación, se observa que la matrona, para proceder a la atención de la paciente, debe realizar una extensión de su extremidad superior al momento de esta para proceder al parto. Es decir, debe contar con un alcance de los brazos al momento de inclinarse (tórax y brazo), abducción de brazos para posicionarse frente a la zona genital de la paciente. Por otro lado, realiza un giro de tronco cada vez que requiere de implementos ubicados en la mesa de parto.

Tabla 8.

Medidas antropométricas de funcionaria.

$\begin{array}{cc}\text { Medida } & \text { Resultado }(\mathrm{cm} .) \\ \text { Altura poplitea } & 45 \\ \text { Altura hombro-asiento } & 57 \\ \text { Altura hombro-suelo } & 113 \\ \text { Altura codo-suelo } & 64 \\ \text { Altura ojo-asiento } & 78 \\ \text { Ojo-paciente } & 80 \\ \text { Altura muslo-asiento } & 25 \\ \text { Ancho caderas } & 50 \\ \text { Distancia glúteo-poplitea } & 60 \\ \text { Alcance funcional } & 90 \\ \text { Ancho mano } & 9\end{array}$

\section{Dimensiones del puesto de trabajo}

Se obtuvieron las dimensiones de la funcionaria evaluada y mobiliario utilizado de la sala de pre-partos/partos, obteniendo lo siguiente: 


\section{Características del asiento}

El asiento utilizado por la funcionaria carece de respaldo para apoyo lumbar, se encuentra desalineado respecto a su horizontal, generando dificultad para situarse en él y permanecer su estancia al momento de la atención de la paciente. Incluso, hay funcionarios que prefieren atender el parto de pie, ya que el asiento no proporciona el confort suficiente para incorporarse.

Tabla 9.

Medidas del asiento.

\begin{tabular}{ccc} 
Medida & \multicolumn{2}{c}{ Resultado (en cm.) } \\
Altura del asiento & Min.: 40 máx.: 68 promedio: 53 \\
Acolchado del asiento & 7 & 38 \\
Profundidad del asiento & 38 \\
\hline
\end{tabular}

Al realizar un análisis en relación a las medidas antropométricas de la funcionaria en comparación a las que presenta el asiento, la altura del asiento se puede regular entre los 40 a $68 \mathrm{~cm}$. y su altura de utilización corresponde a $53 \mathrm{~cm}$; al ser su altura poplítea de 45 $\mathrm{cm}$. quiere decir que el asiento ya se está colocando $8 \mathrm{~cm}$. por sobre su altura poplitea. Por lo tanto, a esa altura ya requiere que ella tenga una elevación de los pies.

\section{Características de la camilla}

La camilla cumple sus funciones correspondientes para su utilización y se ajusta (presenta un mecanismo eléctrico) tanto en altura como inclinación para acomodar a la paciente al momento de tomar la postura de parto (posición ginecológica).

Tabla 10.

Medidas de la camilla.

\begin{tabular}{ccc} 
Medida & \multicolumn{2}{c}{ Resultado (en cm.) } \\
\hline Altura camilla & Min.: 64 & máx.: 100 promedio: 64 \\
Acolchado camilla & 10 \\
Ancho de la camilla & 90 \\
Largo de la camilla & 204 \\
\hline
\end{tabular}

La camilla, al presentar una altura promedio de $64 \mathrm{~cm}$., se ve involucrada a la altura codo-suelo para realizar el trabajo e involucra esfuerzo de la zona lumbar y cervical de la funcionaria, debido a la inclinación e intento para ayudar a la paciente en la extracción del bebé. Además, la altura poplítea (45 cm.) está bajo el nivel de la altura de la cama, por lo cual permite una incorporación eficiente para desarrollar la atención de parto. Por último, tanto la distancia ojo-paciente $(80 \mathrm{~cm}$.), distancia glúteo-poplítea $(60 \mathrm{~cm}$.) $\mathrm{y}$ alcance funcional $(90 \mathrm{~cm}$.) permiten la visualización, incorporación y alcance para la atención de la paciente.

\section{Características de la mesa de partos}

La mesa de parto permite incorporar los implementos utilizados en la atención del parto (paquete de parto e insumos complementarios), su altura y profundidad acogen de buena manera a la funcionaria para retirar los implementos las veces que los requiera.

Tabla 11.

Medidas mesa de parto.

\begin{tabular}{cc} 
Medida & Resultado (en cm.) \\
\hline Altura de la mesa & 80 \\
Ancho de la mesa & 45 \\
Largo de la mesa & 100
\end{tabular}

La altura codo-suelo de la funcionaria evaluada corresponde a 64 cm. en posición sentada, por lo cual está dentro del rango (entre hombro y codo) en relación a la altura de la mesa $(80 \mathrm{~cm})$.

La altura hombro-suelo, al ser de $113 \mathrm{~cm}$., se encuentra dentro del rango óptimo al realizar el esfuerzo, ya que se encuentra sobre los $80 \mathrm{~cm}$. que presenta la altura de la mesa de partos.

Finalmente, al entrevistar a los funcionarios, se identificaron los siguientes trastornos músculo-esqueléticos asociados a la utilización del equipamiento disponible con el que cuenta la unidad de pre-partos/partos:

- Lumbalgias

- Tendinitis

- Tortícolis

Cabe destacar que producto de estas dolencias, los funcionarios deben asistir al médico, utilizar analgésicos, antiinflamatorios y ejercicios kinésicos.

\section{Evaluación de sobrecarga postural}

Por medio del método RULA3, para la evaluación postural de extremidad superior e inferior se encontró sobrecarga postural, confirmando lo observado en la evaluación antropométrica. Los detalles de esta evalaución son los siguientes:

1. El tórax se inclina y se flexiona hacia delante y lateralmente.

2. Se presenta inclinación del brazo y antebrazo.

3. Presencia de aducción y abducción de los brazos para llevar a cabo el trabajo de parto (más de 3 veces por minuto).

En esta situación, al presentar una postura de trabajo de forma sentada para desarrollar la atención, la inexistencia de apoyo lumbar e inestabilidad del asiento utilizado para efectuar el trabajo genera dolencia en la zona lumbar.

Figura 9.

Puntuación nivel 3 RULA. Rediseño de la tarea.

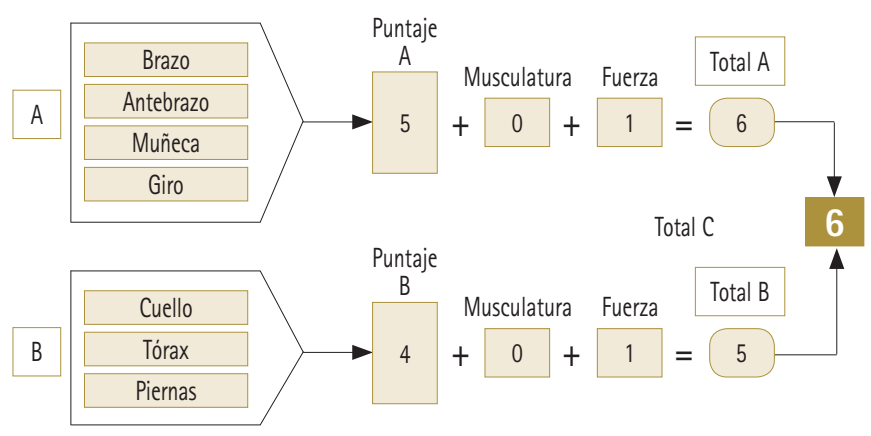

\section{Calidad del sueño}

Análisis de trastornos del sueño y riesgos psicosociales Cuestionario de Pittsburgh ${ }^{4}$

La jornada de trabajo en la unidad de pre-partos/partos se rige por un sistema de cuarto turno, el cual se compone por un turno de día de 12 horas (8:00 a 20:00 hrs) y luego un turno de noche de 12 horas (20:00 a 8:00 hrs.). Posteriormente se accede a dos días libres, para luego seguir en el orden expuesto anteriormente del sistema de trabajo. Al presentar turnos en horario nocturno, donde si se presenta un aumento del flujo de pacientes, el tiempo de descanso se ve reducido o inexistente, por lo que se está predispuesto a padecer algún trastorno del sueño producto de los ciclos circadianos. 
Figura 10.

Atención pre-partos/parto.
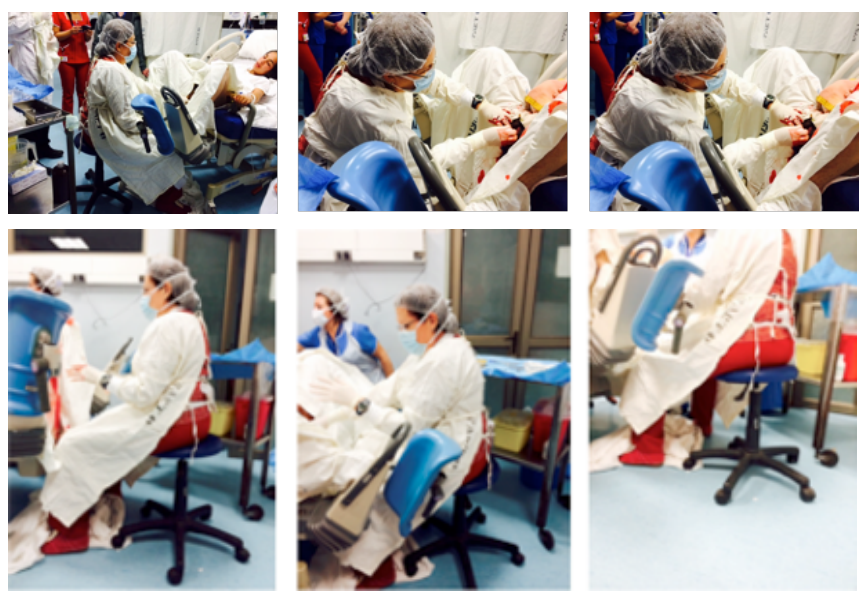

Por este motivo, se analizó a través del cuestionario de Pittsburgh (ver Anexo 1) la calidad del sueño y sus efectos en la funcionaria evaluada, obteniendo los siguientes resultados:

La puntuación total del PSQI corresponde a 12 puntos en la evaluación, obteniendo una calidad de sueño deficiente. El parámetro va en una escala de 0 a 21 puntos (0 indica facilidad para dormir y 21 dificultad severa en todas las siete áreas desarrolladas en el cuestionario). Es decir, se evidencia lo siguiente:

Tabla 12.

Resultados de la evaluación.

$\begin{array}{lc}\text { Item evaluado } & \text { Puntuación } \\ \text { Item 1: Calidad subjetiva del sueño } & 1 \text { punto } \\ \text { Item 2: Latencia de sueño } & 3 \text { puntos } \\ \text { Item 3: Duración del sueño } & 1 \text { punto } \\ \text { Item 4: Eficiencia habitual de sueño } & 3 \text { puntos } \\ \text { Item 5: Perturbaciones del sueño } & 2 \text { puntos } \\ \text { Item 6: Utilización de medicación para dormir. } & 0 \text { puntos } \\ \text { Item 7: Disfunción durante el día } & 2 \text { puntos } \\ \text { Puntuación PSOI TOTAL } & 12 \text { PUNTOS }\end{array}$

Dentro de la evaluación, se evidencian el ítem 2 y 4 con un puntaje de 3 puntos, el cual indica una dificultad severa a nivel de escala (0 indica facilidad, mientras que 3 indica dificultad severa). Es por esto que se observa en el caso de latencia de sueño, que se requiere mayor tiempo para conciliarlo, ya que en el turno el tiempo de descanso depende de la cantidad de trabajo del momento. Además, en eficiencia habitual de sueño se ve distorsionada por el sistema de trabajo en turnos.

Cabe destacar que una de las debilidades del sistema de cuarto turno son su alta irregularidad producto del flujo de pacientes y sus principales fortalezas son el alto número de días libres por semana, moderando el impacto sobre el rito sueño-vigilia, desvinculación social y familiar. ${ }^{6}$

\section{Análisis SUSESO ISTAS 21}

Se realizó la encuesta de riesgos psicosociales SUSESO-ISTAS 215 al turno del equipo de trabajo de la unidad de pre-partos/partos (correspondiente a cuatro matronas, cuatro técnicos en enfermería y un auxiliar de servicio) y se incorporó una psicóloga de apoyo de otra área para obtener un mejor dato de la percepción del turno y la carga mental a la que están sujetos los funcionarios. Se aplicó en su versión breve, la que da antecedentes suficientes a través de sus cinco dimensiones psicosociales, clasificadas en las siguientes tres categorías:

- Bajo: Nivel de exposición psicosocial más favorable para la salud.

- Medio: Nivel de exposición psicosocial intermedio.

- Alto: Nivel de exposición psicosocial más desfavorable para la salud.

Tabla 13.

Resultados encuesta ISTAS 21

\begin{tabular}{|c|c|c|c|c|c|c|c|c|c|c|c|}
\hline $\begin{array}{l}\text { Dimensión } \\
\text { psicosocial } \\
\text { / Cuestionario }\end{array}$ & 1 & 2 & 3 & 4 & 5 & 6 & 7 & 8 & 9 & 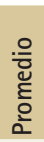 & 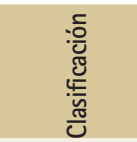 \\
\hline $\begin{array}{l}\text { Exigencias } \\
\text { psicológicas }\end{array}$ & 10 & 12 & 11 & 14 & 14 & 13 & 12 & 11 & 8 & 12 & Desfavorable \\
\hline $\begin{array}{l}\text { Trabajo activo } \\
\text { y desarrollo } \\
\text { de habilidades }\end{array}$ & 3 & 9 & 10 & 11 & 6 & 8 & 13 & 4 & 5 & 8 & Intermedia \\
\hline $\begin{array}{l}\text { Apoyo social } \\
\text { en la empresa } \\
\text { y calidad } \\
\text { de liderazgo }\end{array}$ & 8 & 6 & 13 & 9 & 9 & 8 & 5 & 11 & 5 & 8 & Desfavorable \\
\hline $\begin{array}{l}\text { Dimensión } \\
\text { compensaciones }\end{array}$ & 2 & 7 & 8 & 4 & 5 & 6 & 4 & 8 & 4 & 5 & Intermedia \\
\hline $\begin{array}{l}\text { Dimensión } \\
\text { doble presencia }\end{array}$ & 2 & 8 & 2 & 7 & 2 & 7 & 6 & 8 & 8 & 6 & Desfav \\
\hline
\end{tabular}

De acuerdo a los resultados logrados en la aplicación de esta metodología (Tabla № 7) se observa que en las dimensiones psicosociales más desfavorables corresponden a exigencias psicológicas, apoyo social en la empresa y calidad de liderazgo y dimensión doble presencia, lo que presenta concordancia con los aspectos analizados en el turno, en donde el trabajo no se realiza con tranquilidad de forma constante, ya que va a depender de la cantidad de pacientes y la urgencia que presenten. En relación a las decisiones, estas consisten en jerarquizar el trabajo y mantener una atención constante en él. Sin embargo, ante la presión asistencial, al personal le cuesta mantener el protocolo.

Finalmente, al personal en general le es difícil, por el sistema de turno, dejar de pensar en su situación de vida personal por el horario de trabajo, lo que les impide mantener atención exclusiva a su labor.

\section{Cómo afecta a otras partes del sistema}

En la sala de ingreso con ARO

Cuando las pacientes son referidas a otras unidades se produce conflicto por falta de camas. Las pacientes embarazadas con patologías que van a ARO son derivadas al puerperio cuando no hay camas en esa unidad y ocasionalmente se deben recibir en carro en la unidad de ARO cuando el caso lo amerita. Las pacientes deben ser trasladadas deambulando, en silla de ruedas o en carro dependiendo de la situación de la patología, lo que dificulta, enlentece y produce sobrecarga laboral al auxiliar de ingreso que efectúa la actividad.

\section{En la sala de ingreso con pre-partos/partos}

Las pacientes que ingresan a pre-parto pueden venir deambulando, en silla de ruedas o carro dependiendo de la situación. Aquí el mayor problema es la falta de camas, por lo que la paciente es dejada en carro o se habilita un carro para acostarla. En la medida que van desocupándose camas, se traslada cuando ha iniciado su trabajo de 
parto, lo que es una sobrecarga de trabajo para el personal técnico paramédico que tiene que confeccionar cama y carro. Además, el auxiliar de servicio debe realizar el aseo de las unidades.

\section{En relación al traslado de pacientes}

Hay un incremento de trabajo al aumentar el flujo de pacientes, aumenta por consiguiente la cantidad de traslados.

Falta de personal para realizar los traslados

Debido a que en la actualidad no se cuenta con un número suficiente de personal que lo realice.

\section{CONCLUSIONES}

El hospital de Talca es una organización compuesta por servicios clínicos y servicios de apoyo. Al analizar el sistema general y el subsistema, se puede entender a mayor cabalidad el funcionamiento general y específico de las unidades que componen el subsistema de Obstetricia y ginecología, en las cuales dentro de la evaluación de la unidad de pre-parto/partos se puede concluir:

Se evidencia una carga de trabajo en la matrona, producto del aumento de la cantidad de pacientes que llegan desde ingreso para ser atendidas en su respectiva unidad, lo cual dificulta su atención, enlenteciéndola, por falta de camas disponibles. Por otro lado, en el caso del mobiliario, el piso utilizado para la atención de parto no cumple con las características ergonómicas para desarrollar la labor, debido a la carencia de zonas de apoyo, respaldo y desalineación, lo que produce alteraciones posturales y dolencias en la funcionaria. Sin embargo, la mesa de parto y camilla de atención presentan condiciones favorables para su utilización. Respecto a sobrecarga postural, se evidencia el rediseño de la tarea, la cual debe posibilitar el mejoramiento de la postura de la matrona en la atención del parto.
$\mathrm{Al}$ analizar los trastornos del sueño y riesgos psicosociales se establece, en el caso de la calidad del sueño, un déficit en la latencia y habitualidad del sueño, producidos por el sistema de trabajo en cuarto turno. En el caso de riesgos psicosociales se advierte una preocupación constante del personal por situaciones de presión asistencial, personales y familiares.

Es por esto, que se establecen las siguientes recomendaciones:

- En el subsistema de Obstetricia y Ginecología se puede distinguir en la unidad de pre-parto/partos una sobrecarga de trabajo. Por esto, se sugiere aumentar el personal a 1 matrona más por turno y 1 auxiliar de servicio por turno para mediar el nivel de trabajo.

- Para mejorar el traslado de pacientes se deberá aumentar la cantidad de carros de traslado (actualmente se cuenta con un carro) a 3 unidades en buen estado para facilitar el trayecto, dar seguridad a la paciente y mejorar su desplazamiento.

- Se debe aumentar la cantidad de camas de pre-parto/partos a 2 unidades más y ARO 12 camas más, ya que actualmente se presentan 5 camas en pre-parto y 18 camas en ARO.

- En cuanto a la unidad de pre-parto/partos se debe reestructurar la organización de las dimensiones por unidad de paciente, debido a la falta de privacidad. Por esto se considera 1,5 metros de separación entre una cama y otra.

- Respecto al equipamiento con el que cuenta la unidad de preparto/partos, se debe reconsiderar el cambio del asiento por uno que cuente con zonas de apoyo en la zona lumbar y sea regulable en altura a disposición del funcionario en particular. Con esto, se disminuirá la sobrecarga postural al efectuar la actividad.

- Dentro del trabajo en el turno, se deben repartir las funciones en forma equitativa (controles, atención de partos, tratamientos, controles de signos vitales, monitoreo cardio-fetal, entre otros) y cumplir las indicaciones médicas respectivas para equilibrar la carga de trabajo y fomentar el trabajo en equipo.

\section{REFERENCIAS}

1. Hospital de Talca. Wikipedia [pág web]. [citado 20 may 2015]. Disponible en: http://es.wikipedia.org/wiki/Hospital_de_Talca

2. Bravo C. Manual de organización del servicio de Obstetricia y Ginecología. Hospital Regional de Talca César Garavagno Burotto- Servicio de Salud del Maule. Talca: Hospital Regional de Talca; 2015.

3. Diego-Mas JA. Evaluación postural mediante el método RULA [en línea]. Ergonautas [pág web]. Valencia: Universidad Politécnica de Valencia; 2015 [citado 30 may 2015]. Disponible en: http://www.ergonautas.upv.es/metodos/ rula/rula-ayuda.php.

4. Cuestionario de Pittsburgh de calidad del sueño [en línea]. [Madrid: Elsevier; sd]. [citado 28 may 2015]. Disponible en: http://www.elsevier.es/ficheros/ $\mathrm{mmc} / 27 / 27 \mathrm{v} 46 \mathrm{n} 10 / 27 \mathrm{v} 46 \mathrm{n} 10-90362345 \mathrm{mmc}$. pdf

5. Superintendencia de Seguridad Social. Cuestionario de Evaluación de Riesgos Psicosociales en el Trabajo SUSESO-ISTAS 21; Manual, versión breve. Santiago: Gobierno de Chile; 2013 [citado 28 may 2015]. Disponible en: http://www.ist.cl/ wp-content/uploads/2016/08/MANUAL-breve-ISTAS-11-11-2013.pdf

6. Córdova $V_{1}$ Hevia JC, Figueroa A. Trabajo en turnos en el sector de la salud chileno: una comparación entre el sector público y privado. Cienc Trab [en línea]. 2006 [citado 6 may 2015]; 8(21):147-150. Disponible en: http://www. fucyt.cl/fucytqa/temasinteres/Articulos/Paginas/trabajo-turnos-sector-salud. aspx

Kanawaty G. Estudio de tiempos: Selección y cronometraje del trabajo. En: Kanawaty G. Introducción al estudio de trabajo. $4^{a}$ ed. Ginebra: OIT; 1996. p. 289-304.

Servicio de Salud del Maule-Hospital Regional de Talca. La Historia [en línea]. Talca: Hospital Regional de Talca; 2017 [consultado 20 mar 2017]. Disponible en: http://www.hospitaldetalca.cl/2015/hdetalca/?page_id=3199

Moreno B, Báez C. Factores y riesgos psicosociales, formas, consecuencias, medidas y buenas prácticas [en línea]. Madrid: INSHT; 2010 [consultado 7 may 2015]. Disponible en http://www.insht.es/InshtWeb/Contenidos/Documentacion/ PUBLICACIONES\%2OPROFESIONALES/factores\%20riesgos\%20psico.pdf.

Chile. Ministerio de Salud. Norma Técnica de identificación y evaluación de factores de riesgo de trastornos musculo-esqueléticos relacionados al trabajo (TMERT) de extremidades superiores. Santiago: MINSAL; 2012 [consultado 29 may 2015]. Disponible en: http://web.minsal.cl/portal/url/item/cbb583883dbc1e79e040010165014f3c.pdf. 